05

\title{
Влияние деформации в среде жидкого азота на кинетику десорбции воды из меди и железа при нагревании
}

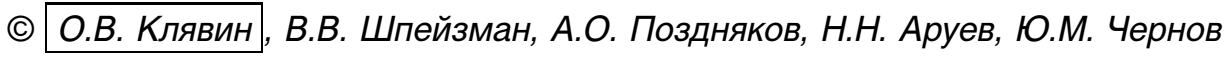 \\ Физико-технический институт им. А.Ф. Иофрфе РАН, \\ 194021 Санкт-Петербург, Россия \\ e-mail: shpeizm.v@mail.ioffe.ru
}

Поступило в Редакцию 23 сентября 2020 г.

В окончательной редакции 27 октября 2020 г.

Принято к публикации 28 октября 2020 г.

На основе представлений о дислокационно-динамической диффузии атомов и молекул среды в приповерхностные слои твердых тел при их деформации рассмотрено влияние предварительной деформации меди и железа в среде жидкого азота, способствующей интенсивному проникновению азота в приповерхностные слои металлов, на положение максимумов зависимости скорости десорбции молекул воды от температуры. Обнаружено смещение максимумов в сторону более высоких температур в результате предварительной деформации. Произведено сравнение различных способов разделения пиков на кривых десорбции воды и оценены энергии активации процессов десорбции в низко- и высокотемпературных областях, а также высказаны предположения о причинах влияния деформации в жидком азоте на характеристики десорбции молекул воды при нагревании.

Ключевые слова: десорбция молекул воды, деформация в среде азота, дислокационно-динамическая диффузия.

DOI: 10.21883/JTF.2021.04.50623.278-20

\section{Введение}

Состоянию молекул воды на поверхности твердых тел посвящено большое число исследований. Многочисленные обзоры и оригинальные теоретические и экспериментальные работы описывают различные аспекты этой проблемы [1-5]. Одним из таких аспектов является открытое и изучаемое в ФТИ им. А.Ф. Иоффе механодинамическое проникновение (МДП) частиц внешней среды в различные типы твердых тел в процессе их пластической деформации. Как показали проведенные исследования, МДП оказывает существенное влияние на десорбцию атомов и молекул среды при последующем нагревании [6,7]. Эффект МДП заключается в аномальной атермической дислокационно-динамической диффузии (ДДД) атомов, молекул и ионов среды в приповерхностный слой материала при пластической деформации. В качестве внедряющихся частиц рассматриваются атомы, молекулы и ионы как непосредственно захваченные из окружающей среды дефектной структурой при ее перестроении, так и частицы, предварительно адсорбированные на поверхности или имплантированные в приповерхностный слой и далее переносимые подвижными дефектами. В [6,7] и более ранних работах было изучено, как влияют на скорость десорбции и общее количество десорбированных при нагревании частиц такие факторы, как вид частиц, величина и способ деформации, тип кристаллической структуры твердого тела, агрегатное состояние и атомно-молекулярный состав среды. Деформация проводилась в основном в среде жидкого или газообразного гелия и азота, а также в воде и воздушной среде. При дальнейшем нагревании в масс-спектрометре следили за десорбцией гелия, азота и воды. Гелий был удобной модельной средой, кроме того, исследования с гелием проводились на оригинальном высокоразрешающем масс-спектрометре с чувствительностью по гелию $10^{9}$ атомов, что позволяло фиксировать малые изменения кинетики его десорбции. Еще одним важным преимуществом гелия как среды было обнаруженное нами его влияние на процесс дробления частиц различных материалов, что позволяло значительно ускорить дробление и получать порошки с размерами в нанометровом диапазоне. Исследования с жидким азотом в качестве среды деформации показало, что он заходит в материал в намного большем количестве, чем другие атомы, молекулы или ионы, находившиеся в среде или адсорбированные поверхностью исходного материала. Так, в [7] показано, что количество азота, выделившегося из железа, деформированного сжатием в жидком азоте до деформации $\varepsilon \sim 35 \%$, может достигать $10^{17} \mathrm{at} / \mathrm{cm}^{2}$ площади поверхности. Это на несколько порядков превышает количество вошедших в материал атомов гелия при деформации в жидком гелии, а также всех других атомов, молекул и ионов, находящихся в среде испытания и на поверхности твердого тела. Поэтому они испытывают жесткую конкуренцию в борьбе с атомами и молекулами азота за ловушки, в которые они могли бы войти в приповерхностные слои в процессе деформации или после нее при нагреве и переносе образца в камеру масс-спектрометра. В настоящем исследовании нас 
интересовала десорбция воды, которая играет важную роль в процессах, проходящих на поверхности, особенно в тонких пленках, подложках и др. [8], и изменение кинетики десорбции после деформации в жидком азоте.

\section{1. Образцы и методика эксперимента}

Эксперименты проводили на образцах монокристаллической меди $(99.99 \% \mathrm{Cu})$ и поликристаллического армко-железа (99.98\% Fe). Монокристаллы меди с ориентацией [110] были выращены методом Бриджмена в графитовых формах в вакууме. Плоские образцы с двумя лопатками и размерами рабочей части $4 \times 10 \times 30 \mathrm{~mm}$ деформировались растяжением в жидком азоте при $T=77 \mathrm{~K}$ на универсальной испытательной машине Instron 1342 со скоростью деформации $\dot{\varepsilon}=10^{-4} \mathrm{~s}^{-1}$. Образцы железа цилиндрической формы с диаметром 3 и высотой $6 \mathrm{~mm}$ вырезались из прутков $14 \times 14 \mathrm{~mm}$, исходных и подвергнутых равноканальному угловому прессованию (РКУП). После 8 проходов РКУП по пути $B c$ (с поворотом на $90^{\circ}$ после каждого прохода) средний размер зерна составлял $\sim 100 \mathrm{~nm}$. Образцы подвергались сжатию при $T=77 \mathrm{~K}$ на машине Instron 1342 со скоростью деформации $\dot{\varepsilon}=10^{-4} \mathrm{~s}^{-1}$.

Десорбция молекул воды из деформированных и недеформированных образцов изучалась с помощью времяпролетного масс-спектрометра MCX-6 с ионизацией частиц электронным ударом при энергиях до $75 \mathrm{eV}$ и выше [7]. Перед исследованиями в масс- спектрометре образцы меди и железа были прокатаны до толщины $0.3 \mathrm{~mm}$. Преимуществом тонких образцов в масс-спектрометрической методике по сравнению с объемными образцами является их большая поверхность, а также возможность более равномерного контролируемого нагрева и достижения за короткое время опыта высоких температур. Были получены кривые десорбции $\mathrm{H}_{2} \mathrm{O}$ при нагревании в области температур $T=20-1200^{\circ} \mathrm{C}$ при постоянной скорости нагревания $120^{\circ} \mathrm{C} / \mathrm{min}$. Ocновной вклад в ошибку измерений давала погрешность измерения температуры, которая составляла $\pm 5^{\circ} \mathrm{C}$. Обработка экспериментальных кривых экстракции молекул $\mathrm{H}_{2} \mathrm{O}$ проводилась двумя способами [9]. В первом способе разделение пиков и оценка вклада каждого из них определялись с использованием нормального закона распределения (по Гауссу). Во втором - путем моделирования каждой стадии процесса решением кинетического уравнения вида $d n / d t=-K \cdot n^{m}$, где $d n / d t-$ скорость десорбции молекул воды, $m$ - порядок реакции, $K-$ константа скорости вида $K=K_{0} \exp (-U / R T)$, $K_{0}$ - предэкспоненциальный множитель, $U-$ энергия активации. В расчетах была использована программа Fitter $^{\mathrm{TM}}[10]$, с помощью которой были получены оценки энергетических параметров кинетических уравнений для разных стадий десорбции воды. Результаты расчетов двумя упомянутыми способами обозначены ниже соответственно $G$ и $F$.

\section{2. Результаты и их обсуждение}

\section{1. Десорбция воды из монокристаллической меди}

Зависимости скорости десорбции воды от температуры для образцов меди без предварительной деформации (тип 1) и после деформации $\varepsilon=14$ (тип 2) и 49.5\% (тип 3) приведены на рис. 1. Там же показаны кривые, полученные в результате разделения пиков двумя описанными выше способами. Экспериментальные результаты в ряде случаев (особенно для образцов без предварительной деформации) имеют участки, на которых скорость десорбции $d n / d t$ возрастает с температурой, а ниспадающий участок отсутствует. Кроме того, десорбция воды не прекращается до конца выбранной области температур. Это затрудняет анализ полученных кривых десорбции. Количественные характеристики пиков десорбции (температура, доля в общем количестве выделившейся при нагревании воды и энергии активации процессов десорбции воды в разных температурных областях) приведены в табл. 1. Как видно из рис. 1 и табл. 1, оба способа выделения пиков имеют как общие черты, так и существенные расхождения по многим показателям. Для первого низкотемпературного пика оба способа обработки дают близкие результаты по температуре максимума: 146 и 142, 199 и 196, 240 и 239 соответственно для образцов типов 1, 2 и 3. Разная форма разложений по нормальному закону и по скорости химических реакций приводит к расхождению долей отдельных пиков в общем количестве десорбированной воды. По этой же причине для лучшего совпадения суммарной кривой распределений третий пик был разбит на два в разложении для образцов типа 1 по нормальному закону ${ }^{1}$.

Несмотря на отмеченные выше трудности анализа кинетики десорбции воды из меди и роли предварительной деформации в среде жидкого азота, можно сделать следующие выводы. В общем случае десорбция воды происходит в три этапа. Первый, низкотемпературный этап, характеризуется $T_{1}=150-250^{\circ} \mathrm{C}$ для всех образцов, второй - для образцов типа 1 и $2-T_{2}=400-700^{\circ} \mathrm{C}$ (для образцов типа 3 этот пик отсутствует при обработке по Гауссу), и высокотемпературный этап с близкими по величине температурами $T_{3}=770-870^{\circ} \mathrm{C}$ (если сравнивать средние значения обоих распределений). Для образцов типа 1 наблюдается существенное различие значений температуры $T_{2}$ и вкладов всех трех полученных пиков в общее количество десорбированной воды при обработке данных разными способами ( $G$

\footnotetext{
${ }^{1}$ Следует заметить, что при разбиении высокотемпературного пика на два оказалось, что доля одного из них крайне мала и составляет всего $3 \%$ (табл. 1). Поэтому при обсуждении результатов он не выделялся как самостоятельный пик, а рассматривался вместе с большим по площади соседним пиком. Подобное рассмотрение представляется оправданным еще и потому, что в разложении по скорости химических реакций малый пик отсутствовал.
} 

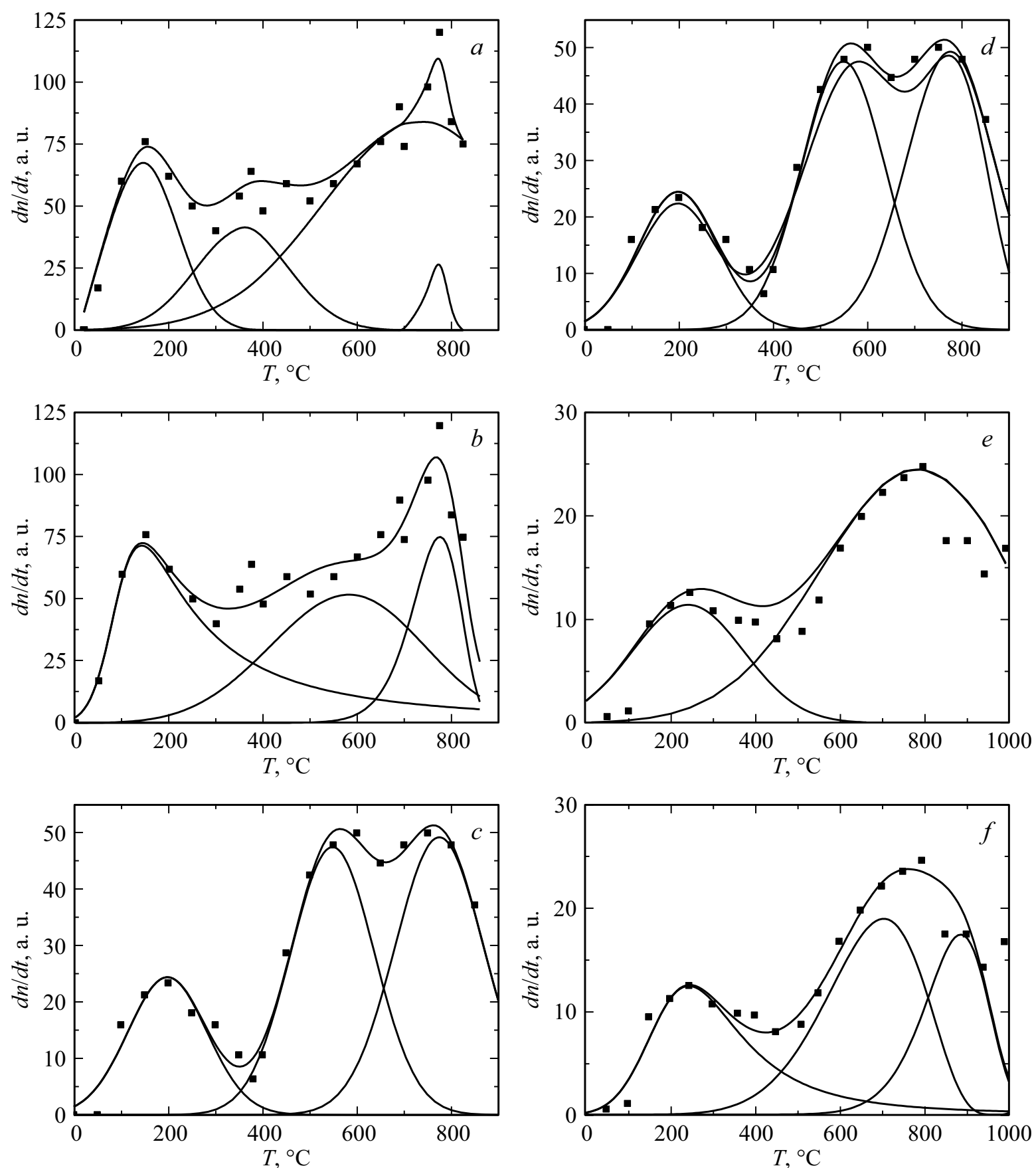

Рис. 1. Зависимость скорости десорбции воды от температуры для меди после прокатки. Предварительная деформация в жидком азоте при $T=77 \mathrm{~K}, \%: 0$ - образцы типа $1(a, b), 14$ - образцы типа $2(c, d)$ и 49.5 - образцы типа $3(e, f)$. Обработка данных: $a, c, e-$ по нормальному закону распределения (по Гауссу), $b, d, f-$ с использованием формул для химических реакций.

и $F$ ). Связано это с трудностями разложения экспериментальной зависимости $d n / d t(T)$, обойти которые удалось путем увеличения порядка реакции при анализе пика $T_{1}$ (рис. $\left.1, b\right)$ и обрыва кривой вблизи максимума $T_{3}$ (рис. $1, a)$.

Из данных табл. 1 следует, что $T_{1}, T_{2}$ и $T_{3}$ возрастают с ростом величины предварительной деформации образцов в жидком азоте. Энергии активации десорбции в общем случае возрастают с номером пика, близость энергий активации для первых двух пиков образцов без предварительной деформации, возможно, связана с упомянутыми выше особенностями обработки результатов. Подобной корреляции энергии активации с величиной предварительной деформации в жидком азоте не было обнаружено. У образцов типа $2(\varepsilon=14 \%)$ энергия активации реакции, полученная для первого пика, оказалась минимальной. Возможно, это связано с особенностями кривой растяжения, описанной в [6] для предварительно 
Таблица 1. Характеристики пиков десорбции воды из меди после предварительной деформации при $T=77 \mathrm{~K}$. Разделение пиков по Гауссу $(G)$ и с использованием кинетического уравнения реакции по программе Fitter $^{\mathrm{TM}}(F)$

\begin{tabular}{|c|c|c|c|c|c|c|c|}
\hline \multirow{5}{*}{$\begin{array}{c}\text { Тип } 1: \varepsilon=0 \\
\text { прокатка от } 3 \\
\text { до } 0.3 \mathrm{~mm}\end{array}$} & \multicolumn{2}{|c|}{$T_{1},{ }^{\circ} \mathrm{C}$} & \multicolumn{2}{|c|}{$T_{2},{ }^{\circ} \mathrm{C}$} & \multicolumn{3}{|c|}{$T_{3},{ }^{\circ} \mathrm{C}$} \\
\hline & \multirow{2}{*}{$\begin{array}{c}G \\
146 \\
\end{array}$} & \multirow{2}{*}{$\begin{array}{c}F \\
142\end{array}$} & \multirow{2}{*}{$\frac{G}{359}$} & \multirow{2}{*}{$\frac{F}{575}$} & \multicolumn{2}{|c|}{$G$} & \multirow{2}{*}{$\begin{array}{c}F \\
775\end{array}$} \\
\hline & & & & & 737 & 772 & \\
\hline & \multicolumn{2}{|c|}{ Доля пика 1, \% } & \multicolumn{2}{|c|}{ Доля пика 2, \% } & \multicolumn{3}{|c|}{ Доля пика 3, \% } \\
\hline & 23 & 42 & 19 & 39 & 55 & 3 & 19 \\
\hline$E, \mathrm{~kJ} / \mathrm{mol}(\mathrm{eV})$ & & $33(0.34)$ & & $30(0.31)$ & & & $170(1.76)$ \\
\hline \multirow{5}{*}{$\begin{array}{c}\text { Тип 2: } \varepsilon=14 \% \\
\text { в жидком азоте } \\
+ \text { прокатка от } 3 \\
\text { до } 0.3 \mathrm{~mm}\end{array}$} & \multicolumn{2}{|c|}{$T_{1},{ }^{\circ} \mathrm{C}$} & \multicolumn{2}{|c|}{$T_{2},{ }^{\circ} \mathrm{C}$} & \multicolumn{3}{|c|}{$T_{3},{ }^{\circ} \mathrm{C}$} \\
\hline & $G$ & $F$ & G & $F$ & \multicolumn{2}{|c|}{ G } & $F$ \\
\hline & 199 & 196 & 550 & 547 & \multicolumn{2}{|c|}{774} & 775 \\
\hline & \multicolumn{2}{|c|}{ Доля пика 1, \% } & \multicolumn{2}{|c|}{ Доля пика 2, \% } & \multicolumn{3}{|c|}{ Доля пика 3, \% } \\
\hline & 19 & 18 & 39 & 41 & & & 41 \\
\hline$E, \mathrm{~kJ} / \mathrm{mol}(\mathrm{eV})$ & & $19(0.19)$ & & $53(0.55)$ & & & $104(1.08)$ \\
\hline \multirow{5}{*}{$\begin{array}{c}\text { Тип 3: } \varepsilon=49.5 \% \\
\text { в жидком азоте } \\
+ \text { прокатка от } 3 \\
\text { до } 0.3 \mathrm{~mm}\end{array}$} & \multicolumn{2}{|c|}{$T_{1},{ }^{\circ} \mathrm{C}$} & \multicolumn{2}{|c|}{$T_{2},{ }^{\circ} \mathrm{C}$} & \multicolumn{3}{|c|}{$T_{3},{ }^{\circ} \mathrm{C}$} \\
\hline & $G$ & $F$ & $G$ & $F$ & \multicolumn{2}{|c|}{$G$} & $F$ \\
\hline & 240 & 239 & - & 706 & \multicolumn{2}{|c|}{780} & 874 \\
\hline & \multicolumn{2}{|c|}{ Доля пика 1, \% } & \multicolumn{2}{|c|}{ Доля пика 2, \% } & \multicolumn{3}{|c|}{ Доля пика 3, \% } \\
\hline & 29 & 32 & - & 42 & 71 & 26 & \\
\hline$E, \mathrm{~kJ} / \mathrm{mol}(\mathrm{eV})$ & & $25(0.26)$ & & $54(0.56)$ & & & $141(1.46)$ \\
\hline
\end{tabular}

деформированной (наноструктурной) меди. При малых деформациях меди в жидком гелии пластическое течение осуществляется за счет внутризеренного скольжения дислокаций, в области $5<\varepsilon<14 \%$ - путем двойникования, а при $\varepsilon>14 \%$ после исчерпания согласованных переориентаций решетки за счет ее механического двойникования инициируется поворотный (дисклинационный) механизм деформации. Сравнение с подобными образцами представляется оправданным, поскольку в нашем случае все образцы перед масс-спектрометрическими исследованиями подвергались интенсивной прокатке. Таким образом, энергетические характеристики ловушек атомов и молекул среды могут зависеть не только от величины, но и от механизма деформации.

\section{2. Десорбция воды из крупнозернистого (КЗ) и ультрамелкозернистого (УМЗ) армко-железа}

На рис. 2 приведены кривые десорбции воды из К3 железа без предварительной деформации (тип 1) и после деформации в жидком азоте (тип 2). Аналогичные кривые для УМЗ железа показаны на рис. 3. Величина предварительной деформации и в том, и в другом случаях была близкой: $36 \%$ для КЗ железа и 31\% для УМЗ. Результаты, полученные разделением пиков, и их вклады в общее количество десорбированной воды приведены в табл. 2 и 3. Так же, как и для меди, рассматривались два способа обработки результатов: по нормальному закону распределения $(G)$ и по уравнению для скоростей реакций $(F)$. Во всех случаях независимо от структуры материала с предварительной деформацией или без нее, а также способа обработки результатов, четко разделяются две области: низко- и высокотемпературная. Иногда при описании десорбции воды в какой-либо температурной области с целью лучшего совпадения с экспериментом использовалось разложение с двумя максимумами (см. КЗ, высокотемпературный пик, оба типа образцов, разложение $G$; УМЗ, образцы типа 1, оба пика, разложение $G$ и низкотемпературный пик, разложение $F$ ). Характерные температуры пиков: $T_{1}=400-480^{\circ} \mathrm{C}$ и $T_{2}=800-1100^{\circ} \mathrm{C}$.

Для образцов КЗ железа влияние предварительной деформации в жидком азоте приводит к небольшому повышению температуры обоих пиков, более заметное для высокотемпературного пика, и к уменьшению доли первого пика в общем количестве десорбированной воды. Повышение температуры пиков после предварительной деформации наблюдается и у УМЗ железа. Температуры пиков лежат в области $T_{1}=400-500^{\circ} \mathrm{C}$ и $T_{2}=850-1100^{\circ} \mathrm{C}$. У недеформированного в жидком 

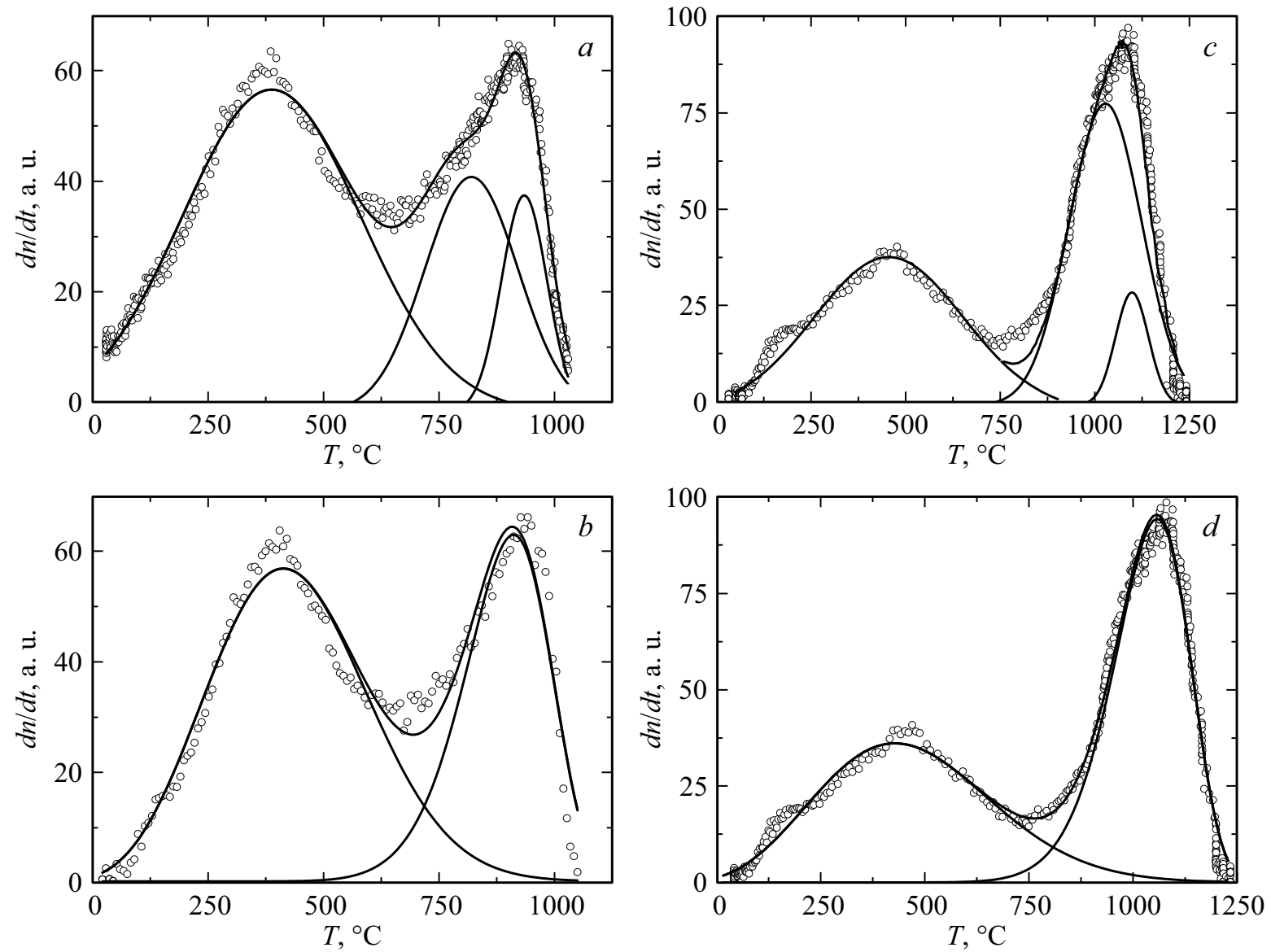

Рис. 2. Зависимость скорости десорбции воды от температуры из: $a, b-$ прокатанного (образцы типа 1 ) и $c, d-$ после предварительной деформации $\varepsilon=36 \%$ в жидком азоте с последующей прокаткой (образцы типа 2) армко-железа. Обработка данных: $a, c-$ с использованием нормального закона распределения (по Гауссу) и $b, d-$ по формулам для химических реакций.

Таблица 2. Характеристики пиков десорбции воды из прокатанного и после деформации $36 \%$ в жидком азоте с последующей прокаткой КЗ армко-железа. Разделение пиков по Гауссу $(G)$ и с использованием кинетического уравнения реакции по программе Fitter $^{\mathrm{TM}}(F)$

\begin{tabular}{|c|c|c|c|c|c|}
\hline \multirow{5}{*}{$\begin{array}{c}\text { Тип } 1: \varepsilon=0 \\
+ \text { прокатка } \\
\text { от } 3 \text { до } \\
0.3 \mathrm{~mm}\end{array}$} & \multicolumn{2}{|c|}{$T_{1}, T_{2},{ }^{\circ} \mathrm{C}$} & \multicolumn{3}{|c|}{$T_{2}, T_{2},{ }^{\circ} \mathrm{C}$} \\
\hline & \multirow{2}{*}{$\frac{G}{397}$} & \multirow{2}{*}{$\begin{array}{c}F \\
412\end{array}$} & \multicolumn{2}{|c|}{$G$} & \multirow{2}{*}{$\frac{F}{942}$} \\
\hline & & & 812 & 912 & \\
\hline & \multicolumn{2}{|c|}{ Доля пика 1, \% } & \multicolumn{3}{|c|}{ Доля пика 2, \% } \\
\hline & 58 & 64 & 28 & 14 & 36 \\
\hline$E, \mathrm{~kJ} / \mathrm{mol}$ & & 16 & & & 113 \\
\hline \multirow{5}{*}{$\begin{array}{c}\text { Тип 2: } \varepsilon=36 \% \\
\text { в жидком } \\
\text { азоте + } \\
\text { прокатка от } \\
3 \text { до } 0.3 \mathrm{~mm}\end{array}$} & \multicolumn{2}{|c|}{$T_{1}, T_{2},{ }^{\circ} \mathrm{C}$} & \multicolumn{3}{|c|}{$T_{2}, T_{2},{ }^{\circ} \mathrm{C}$} \\
\hline & $G$ & $F$ & \multicolumn{2}{|c|}{$G$} & $F$ \\
\hline & 457 & 430 & 1026 & 1097 & 1057 \\
\hline & \multicolumn{2}{|c|}{ Доля пика 1, \% } & \multicolumn{3}{|c|}{ Доля пика 2, \% } \\
\hline & 49 & 46 & 43 & 8 & 54 \\
\hline$E, \mathrm{~kJ} / \mathrm{mol}$ & & 14 & & & 149 \\
\hline
\end{tabular}



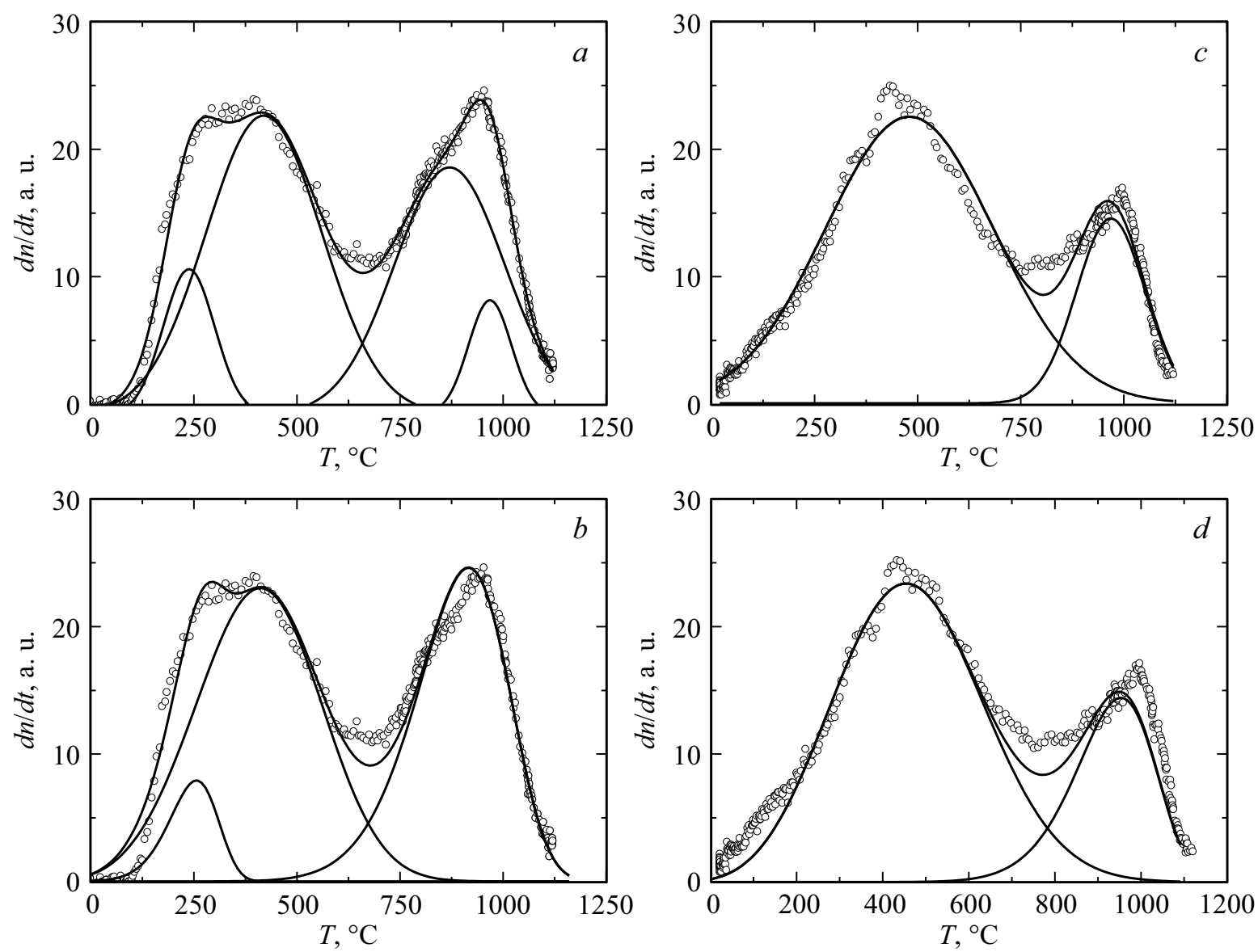

Рис. 3. Зависимость скорости десорбции воды от температуры из: $a, b-$ прокатанного (образцы типа 1 ) и $c, d-$ после предварительной деформации $\varepsilon=31 \%$ в жидком азоте с последующей прокаткой (образцы типа 2) УМЗ (после РКУП) армкожелеза. Обработка данных: $a, c-\mathrm{c}$ использованием нормального закона распределения (по Гауссу) и $b, d-$ по формулам для химических реакций.

Таблица 3. Характеристики пиков десорбции воды из прокатанного и после деформации $31 \%$ в жидком азоте с последующей прокаткой УКЗ армко-железа. Разделение пиков по Гауссу $(G)$ и с использованием кинетического уравнения реакции по программе $\operatorname{Fitter}^{\mathrm{TM}}(F)$

\begin{tabular}{|c|c|c|c|c|c|c|c|}
\hline \multirow{5}{*}{$\begin{array}{c}\text { Тип 1: } \varepsilon=0 \\
+ \text { прокатка от } \\
3 \text { до } 0.3 \mathrm{~mm}\end{array}$} & \multicolumn{2}{|c|}{$T_{1 a}, T_{2},{ }^{\circ} \mathrm{C}$} & \multicolumn{2}{|c|}{$T_{1 b}, T_{2},{ }^{\circ} \mathrm{C}$} & \multicolumn{3}{|c|}{$T_{2}, T_{2},{ }^{\circ} \mathrm{C}$} \\
\hline & $G$ & $F$ & $G$ & $F$ & \multicolumn{2}{|c|}{$G$} & $F$ \\
\hline & 237 & 254 & 419 & 410 & 869 & 967 & 913 \\
\hline & \multicolumn{2}{|c|}{ Доля пика 1 a, \% } & \multicolumn{2}{|c|}{ Доля пика 1 b, \% } & \multicolumn{3}{|c|}{ Доля пика 2, \% } \\
\hline & 10 & 7 & 47 & 25 & 37 & 6 & 41 \\
\hline$E, \mathrm{~kJ} / \mathrm{mol}$ & & 58 & & 31 & & & 122 \\
\hline \multirow{5}{*}{$\begin{array}{c}\text { Тип 2: } \varepsilon=31 \% \\
\text { в жидком } \\
\text { азоте }+ \\
\text { прокатка } \\
\text { от } 3 \text { до } 0.3 \mathrm{~mm}\end{array}$} & \multicolumn{3}{|c|}{$T_{1}, T_{2},{ }^{\circ} \mathrm{C}$} & \multicolumn{4}{|c|}{$T_{2}, T_{2},{ }^{\circ} \mathrm{C}$} \\
\hline & \multicolumn{2}{|c|}{$G$} & $F$ & \multicolumn{2}{|c|}{$G$} & \multicolumn{2}{|c|}{$F$} \\
\hline & \multicolumn{2}{|c|}{480} & 450 & \multicolumn{2}{|c|}{967} & \multicolumn{2}{|c|}{950} \\
\hline & \multicolumn{3}{|c|}{ Доля пика 1, \% } & \multicolumn{4}{|c|}{ Доля пика 2, \% } \\
\hline & \multicolumn{2}{|c|}{79} & 75 & \multicolumn{2}{|c|}{21} & \multicolumn{2}{|c|}{25} \\
\hline$E, \mathrm{~kJ} / \mathrm{mol}$ & & & 20 & & & & \\
\hline
\end{tabular}


азоте УМЗ железа появляется небольшой пик в области $250^{\circ} \mathrm{C}$ с неожиданно высокой энергией активации.

Условия десорбции воды в описанных выше экспериментах существенно отличаются от свободного выхода молекул воды. Десорбция воды искусственно затруднена большим количеством атомов и молекул азота, вошедших в приповерхностный слой материала при предварительной деформации в среде жидкого азота. Возможно, поэтому не обнаружен пик при $140-180^{\circ} \mathrm{C}$ на железе, который наблюдали в [11]. Небольшое отклонение экспериментальных данных от расчетных в этой области температур можно трактовать как наличие малого количества слабосвязанной воды также и в нашем случае. Несовпадение энергий активации, полученные в настоящей работе и в [11], можно объяснить тем, что молекулы воды связаны с поверхностью железа через молекулы азота.

\section{Заключение}

Взаимодействие воды с твердыми поверхностями играет центральную роль в различных явлениях в природе и деятельности человека, таких как электрохимические процессы в водном растворе, коррозия, разрушение горных пород и др. $[8,12,13]$. Представляет интерес она и для ряда космологических проблем, например, для образования частиц льда в стратосфере и химии межзвездных процессов [14]. В течение последних лет адсорбция воды на поверхностях твердых тел, преимущественно монокристаллических металлов различной ориентации, интенсивно исследовалась в лабораториях с помощью разнообразных экспериментальных методик. Было установлено, что на поверхности благородных металлов (золото, серебро, а также платина и другие металлы платиновой группы - рутений, родий, палладий, осмий, иридий) вода сохраняет молекулярную форму. На других металлах (хром, железо, молибден, ванадий) вода адсорбируется в диссоциированном виде, как $\mathrm{H}^{+}$ и $\mathrm{OH}^{-}$, а кобальт, никель, медь, вольфрам образуют промежуточную группу [12-16]. Кроме молекулярной и диссоциированной воды в зависимости от вида поверхности и условий эксперимента вода образует различные низкоразмерные структуры, начиная от изолированных мономеров и кластеров до одномерных и двумерных цепочек [8]. Большинство из перечисленных выше результатов получены методами компьютерного моделирования для идеальных поверхностей и присутствия воды как единственного адсорбента. В реальных условиях картина взаимодействия атомов и молекул среды с поверхностью материалов представляется значительно более сложной. Модель дислокационно-динамической диффузии (ДДД) позволяет связать явление адсорбции атомов и молекул среды с дефектной структурой кристаллических тел. В [9] нами было продемонстрировано влияние среды гелия при диспергировании частиц цемента и деформации алюминия при температуре $4.2 \mathrm{~K}$ на процесс десорбции воды. В настоящей работе с позиций ДДД показано на примере меди и железа, что деформация в среде жидкого азота также влияет на десорбцию воды с поверхности и из приповерхностных слоев материалов. При деформации материала или его разрушении (дроблении частиц) атомы и молекулы среды активно входят в приповерхностные слои материала, тем самым изменяя характеристики десорбции всех адсорбентов, в том числе воды как одного из важнейших из них. В зависимости от вида среды деформации происходит перераспределение по различного рода ловушкам уже имевшихся в материале и вновь вошедших атомов и молекул среды, что приводит к изменению физических и химических свойств поверхности и приповерхностных слоев материалов.

\section{Конфликт интересов}

Авторы заявляют, что у них нет конфликта интересов.

\section{Список литературы}

[1] H.J. Feibelman. Phys. Today, 63, 34 (2010).

[2] S. Gim, K.J. Cho, H. Lim, H. Kim. Sci. Rep., 9, 14805 (2019). https://doi.org/10.1038/s41598-019-51323-5

[3] O. Björneholm, M.H. Hansen, A. Hodgson, L.M. Liu, D.T. Limmer, A. Michaelides, P. Pedevilla, J. Rossmeisl, H. Shen, G. Tocci, E. Tyrode, M.M. Walz, J. Werner, H. Bluhm. Chem. Rev., 116, 7698 (2016).

[4] L. Blumenthal, J.M. Kahk, R. Sundararaman, P. Tangney, J. Lischner. RSC Adv., 7, 43660 (2017).

[5] Z. Guo, F. Ambrosio, W. Chen, P. Gono, A. Pasquarello. Chem. Mater., 30, 94 (2018).

[6] О.В. Клявин, В.И. Николаев, Л.В. Хабарин, Ю.М. Чернов, В.В. Шпейзман. ФТТ, 45, 2187 (2003).

[7] О.В. Клявин, В.И. Николаев, О.Ф. Поздняков, Б.И. Смирнов, Ю.М. Чернов, В.В. Шпейзман. ФТТ, 52, 2336 (2010).

[8] S. Meng, E.G. Wang, S. Gao. Phys. Rev., 69, 19544 (2004).

[9] О.В. Клявин, Н.Н. Аруев, А.О. Поздняков, Ю.М. Чернов, В.В. Шпейзман. ЖТФ, 90, 238, 2020.

[10] A.L. Pomerantsev, O.Ye. Rodionova. Chem. Intell. Lab. Syst., 79 (1-2), 73 (2005).

[11] В.Н. Корсаков, О.Ф. Поздняков, Н.З. Евтюков, А.Д. Яковлев, Ю.А. Ремизов. Лакокрасочные материалы и их применение, 5, 37 (1984).

[12] W. Lew, M.C. Crowe, E. Karp, C.T. Campbell. J. Phys. Chem. C, 115, 9164 (2011).

[13] А.М. Кузнецов. Соросовский образовательный журнал, $6(5), 45$ (2000). http://www.pereplet.ru/nauka/Soros/pdf/0005_045.pdf

[14] D. Lowe, A.R. Mac Kenzie. J. Atmos. Solar-Terrestrial Phys., 70 (1), 13 (2008).

[15] J. Carrasco, J. Klimes, A. Michaelides. J. Chem. Phys., 138, 024708 (2013). https://doi.org/10.1063/1.4773901

[16] A. Ignaczak, J. Gomes. J. Electroanal. Chem., 240, 209 (1997). 